\title{
Whole tumor RNA-sequencing and deconvolution reveal a clinically-prognostic PTEN/PI3K-regulated glioma transcriptional signature
}

\author{
Yuan Pan ${ }^{1}$, Erin C. Bush ${ }^{2}$, Joseph A. Toonen ${ }^{1}$, Yu Ma ${ }^{1}$, Anne C. Solga ${ }^{1}$, Peter A. \\ Sims $^{2}$ and David H. Gutmann ${ }^{1}$ \\ ${ }^{1}$ Department of Neurology, Washington University School of Medicine, St. Louis, MO, USA \\ ${ }^{2}$ Departments of Systems Biology and of Biochemistry and Molecular Biophysics, Columbia University Medical Center, New \\ York, NY, USA
}

Correspondence to: David H. Gutmann, email: gutmannd@wustl.edu

Peter A. Sims, email: pas2182@cumc.columbia.edu

Keywords: astrocytoma, neurofibromatosis, AKT, glioma stem cell, microglia

Received: February 10, 2017 Accepted: March 30, $2017 \quad$ Published: April 18, 2017

Copyright: Pan et al. This is an open-access article distributed under the terms of the Creative Commons Attribution License 3.0 (CC BY

3.0), which permits unrestricted use, distribution, and reproduction in any medium, provided the original author and source are credited.

\section{ABSTRACT}

The concept that solid tumors are maintained by a productive interplay between neoplastic and non-neoplastic elements has gained traction with the demonstration that stromal fibroblasts and immune system cells dictate cancer development and progression. While less studied, brain tumor (glioma) biology is likewise influenced by non-neoplastic immune system cells (macrophages and microglia) which interact with neoplastic glioma cells to create a unique physiological state (glioma ecosystem) distinct from that found in the normal tissue. To explore this neoplastic ground state, we leveraged several preclinical mouse models of neurofibromatosis type 1 (NF1) optic glioma, a low-grade astrocytoma whose formation and maintenance requires productive interactions between non-neoplastic and neoplastic cells, and employed whole tumor RNA-sequencing and mathematical deconvolution strategies to characterize this low-grade glioma ecosystem as an aggregate of cellular and acellular elements. Using this approach, we demonstrate that optic gliomas generated by altering the germline $\mathrm{Nf1}$ gene mutation, the glioma cell of origin, or the presence of co-existing genetic alterations represent molecularlydistinct tumors. However, these optic glioma tumors share a 25-gene core signature, not found in normal optic nerve, that is normalized by microglia inhibition (minocycline), but not conventional (carboplatin) or molecularly-targeted (rapamycin) chemotherapy. Lastly, we identify a genetic signature conferred by Pten reduction and corrected by PI3K inhibition. This signature predicts progression-free survival in patients with either low-grade or high-grade glioma. Collectively, these findings support the concept that gliomas are composite ecological systems whose biology and response to therapy may be best defined by examining the tumor as a whole.

\section{INTRODUCTION}

Histological examination of most solid tumors reveals that these cancers are composed of numerous distinct cell types, which are embedded in a rich extracellular matrix containing growth factors and cytokines. Among the cell types in these tumors are differentiated and stem-like neoplastic cells, as well as non-neoplastic cells, such as monocytes, fibroblasts, and endothelial cells [1]. With the recognition that the biological behaviors of many solid cancers are dictated by the productive interactions between these diverse cellular and acellular elements, the concept of a cancer ecosystem has been advanced [2-4]. In support of this concept, non-neoplastic stromal cells, such as fibroblasts and immune system cells, have been shown to drive solid tumor development and progression [5, 6]. Within the central nervous system, brain tumor (glioma) growth in experimental mouse models is similarly controlled by specific monocyte populations, including microglia and macrophages, that are recruited by the tumor cells through the elaboration of chemokines, like colony stimulating 
factor 1 (CSF-1) [7]. Additionally, productive interactions between monocytes and glioma cells create a feed-forward paracrine circuit that perpetuates high-grade glioma growth [8-10]. These studies in high-grade malignancies have suggested alternative approaches to cancer treatment, in which targeted therapies against stromal cell types or signals might emerge as a potential adjuvant approaches [11-15].

One of the clinical situations where stromal therapies might be particularly useful is in the treatment of pediatric low-grade tumors. Unlike adult high-grade malignancies, these tumors are slow growing (with proliferative indices often less than 1\%) and arise in children whose brains are still developing. Therapies that target unique elements of the tumor ecosystem, not shared by the developing brain, would be highly desirable. This issue is well illustrated by the Neurofibromatosis type 1 (NF1) cancer predisposition syndrome, in which affected children are prone to the formation of low-grade nervous system tumors (peripheral nerve sheath tumors and optic pathway gliomas). Examination of these tumors reveals prominent immune system infiltration and interdependencies between neoplastic and non-neoplastic cell types [16-22]. Specifically, the formation and continued growth (maintenance) of low-grade optic gliomas in mice require a supportive tumor microenvironment containing microglia, which are attracted to the developing cancer by chemokines [20], and elaborate specific growth factors that promote neoplastic cell growth [19]. These cellular interactions could be hypothesized to create a new homeostatic state, not present in the non-neoplastic optic nerve, and thus establish new circuitries and interdependencies for chemotherapeutic targeting.

In the current study, we employed whole-tumor RNA-sequencing and mathematical deconvolution strategies using preclinical mouse models of $N f 1$ optic glioma to characterize the tumor ecosystem as an aggregate gene expression pattern distinct from the healthy optic nerve. Leveraging numerous different murine optic glioma genetically-engineered mouse (GEM) models, we found that histologically-similar tumors are molecularly distinct. However, despite their uniqueness, all $N f 1$ murine optic gliomas examined share a 25 -gene expression signature that distinguishes tumor tissue from normal optic nerve. This core genetic signature was minimally affected by conventional (carboplatin) or biologically-targeted (rapamycin) therapy, but was normalized following minocycline treatment, which inhibits tumor-associated microglia function. Lastly, we discovered a PTEN/PI3Kdriven molecular signature that correlates with the overall survival of patients with either low-grade or high-grade glioma. Taken together, our study suggests that shared and unique whole-tumor gene expression patterns may serve as biomarkers of tumor response to therapy and patient survival.

\section{RESULTS AND DISCUSSION}

\section{Whole tumor RNA-sequencing of a diverse collection of $\boldsymbol{N f 1}$ optic glioma tumors reveals molecularly-distinct neoplasms}

In an effort to accurately represent the heterogeneity inherent in human NF1-associated optic pathway glioma (NF1-OPG), we have previously engineered mice that differ with respect to the germline $N f 1$ gene mutation (null allele (FMC) [23] versus NF1 patient germline NF1 gene mutation; R681* (F18C) [24]), the cell of origin $\left(\mathrm{GFAP}^{+}\right.$neuroglial progenitors (FMC) [23] versus Olig2 ${ }^{+}$progenitor cells (FMOC) (Solga A, manuscript in preparation)), and the presence of a co-existing heterozygous Pten deletion in the tumor cells (FMPC) $[25,26]$. Using these four optic glioma strains, we performed whole tumor RNA-Seq on 27 specimens along with four normal age- and sex-matched optic nerves. Gene expression heatmap representation of all 31 samples across 4,624 genes revealed differentially-expressed gene sets in at least one pairwise comparison relative to normal optic nerve, as well as between NfI GEM optic glioma models (Figure 1A). While some sample-to-sample heterogeneity was found between whole tumor specimens from the same GEM model, significantly larger differences among the four models were demonstrated by the average profiles (Figure 1B). Because these expression profiles were from homogenized whole tumor tissue, the observed molecular differences between models likely reflect contributions from multiple distinct cell types. However, despite clear differences in tumor latency, proliferative rates, and tumor volumes, immunohistochemical analyses of these tumors revealed only slight differences in the percentages of microglia ( $\mathrm{Iba}^{+}$cells), astroglial $\left(\mathrm{GFAP}^{+}\right.$or $\mathrm{S} 100 \beta^{+}$cells), or Olig2 ${ }^{+}$progenitor cells [23-25].

To better understand the murine $N f 1$ optic glioma ecosystem as a whole, we next sought to estimate the cell type-specific contributions to the observed gene expression differences. In previous studies, we and others have used computational approaches to deconvolve the data into potential cell type profiles. Whole transcriptome deconvolution requires two basic inputs - genomewide transcript abundances and a measure of cellular composition - both of which can be derived from RNA-Seq data. As demonstrated in our previous study, in which we applied deconvolution to human high-grade gliomas, we can use the abundance of a cell type-specific marker or set of markers for each cell type in any given tissue to estimate the fractional composition of each cell type [27, 28]. Hence, we are able to use the RNA expression levels of cell type-specific marker genes in the composite profiles of each specimen to solve for an estimated abundance of each gene in each cell type by least-squares regression. While this approach assumes that cell type-specific RNA 
abundances are invariant across specimens, the fitting procedure allows us to restrict our analysis to genes with expression patterns that are fit well by this model.

For these analyses, we specifically focused on glial cell populations (microglia and macroglia [oligodendrocytes, astrocytes, oligodendrocyte precursors]; Figure 1C-1F). As such, the glial cell typespecific contributions for genes that are differentially expressed $\left(p_{\text {adj }}<0.01, \mid \log _{2}\right.$ (fold-change $) \mid>1$ ) between each Nf1 GEM optic glioma model and normal optic nerve can be represented, as estimated by computational deconvolution. Using this approach, several important observations were made: First, all of the Nfl GEM optic glioma models showed significant depletion of genes specific to mature oligodendrocytes compared to normal optic nerve (FF). Second, while all four models exhibited enrichment of oligodendrocyte progenitor cell (OPC) gene expression, this finding was most pronounced in the FMOC and FMC optic glioma models relative to the F18C and FMPC optic glioma models. Third, the largest distinction between the FMPC optic glioma model and the normal optic nerve was enrichment of astrocyte gene expression. This result suggests that Pten reduction, which is the only genetically-engineered difference between the FMC and FMPC optic glioma models, may alter the glial lineage character of the tumor. However, these changes in gene expression did not correlate with changes in Olig2 ${ }^{+}$, $\mathrm{NG}^{+}$or $\mathrm{GFAP}^{+}$cell numbers between $N f 1$ GEM optic glioma strains ([24, 25]; Supplementary Figure 1). The apparent disconnect between glial cell-estimated gene expression and actual glial cell numbers suggests that these differentially-expressed gene signatures reflect global changes in the tumor ecosystem, rather than alterations in overall tumor cellular composition. Moreover, analysis of previously-acquired RNA-sequencing data from FMC tumor-associated microglia relative to those from $\mathrm{Nfl+/}$ mice [19] demonstrated that the differentially-expressed genes assigned to microglia by deconvolution were not similarly altered in the actual microglia from these tumors (data not shown). Collectively, these results support the notion that the gene expression patterns in the tumor do not reflect the individual contributions of each cell type, but rather the composite interactions of all cell types.

\section{All $N f 1$ optic glioma GEM models share a core gene expression signature that is minocycline- sensitive}

While Figure 1 highlights the molecular differences among the four different $N f 1$ optic glioma models, all of
C

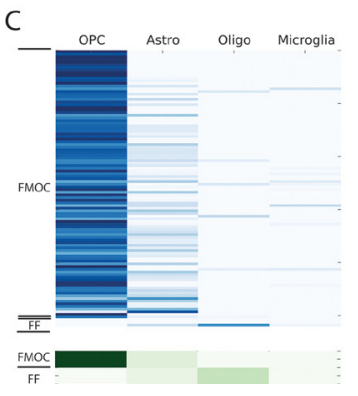

FMOC
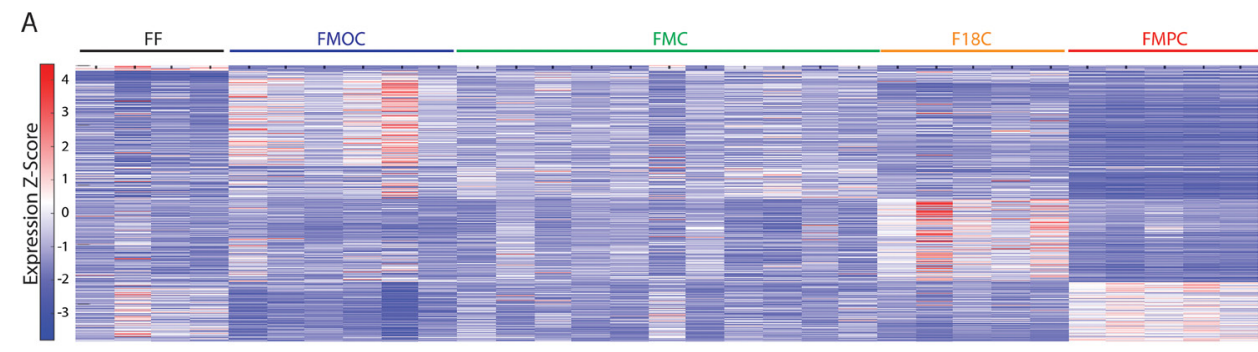

FMC
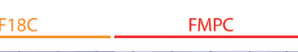

B FF FMOC FMC F18C FMPC

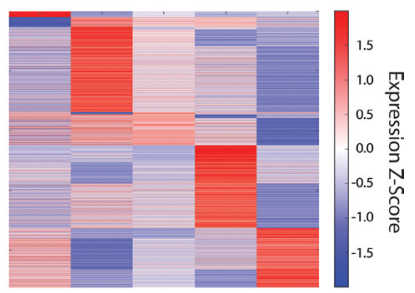

$\mathrm{D}$
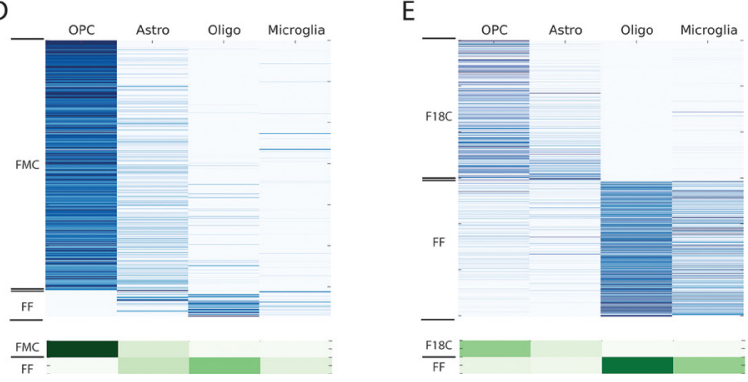

F

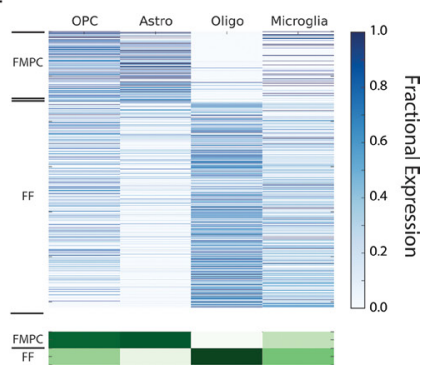

Figure 1: (A) Heatmap of RNA-Seq expression z-scores computed for all genes that are differentially expressed ( $\mathrm{p}_{\text {adj }}<0.01, \mid \log _{2}($ foldchange $\mid>1$ ) between all pairwise comparisons of the optic glioma models and the healthy optic nerve (FF) controls. Each column in the heatmap is an individual sample. (B) Same as (A), but averaged over all samples for each genotype. (C) Computational deconvolution of cell type-specific gene expression for genes that are differentially expressed between FMOC tumors and FF controls. In the top panel, each row in the heatmap corresponds to a specific differentially-expressed gene and each column corresponds to a cell type. The lower panel shows the aggregate expression in each cell type for all genes that are high in FMOC or FF tumors. The analysis shows that the FMOC tumors are highly enriched in OPC-like gene expression. (D) Same as (C), but for differentially-expressed genes from the comparison of FMC tumors to FF controls. Similar to FMOC, the FMC tumors are also dominated by an OPC-like gene expression pattern. (E) Same as (C), but for differentially-expressed genes from the comparison of F18C tumors to FF controls. The F18C tumors have both higher OPClike and astrocytic gene expression relative to FF controls. (F) Same as (C), but for differentially-expressed genes from the comparison of FMPC tumors to FF controls, demonstrating a more pronounced astrocytic gene expression in FMPC gliomas. OPC, oligodendrocyte progenitor cell; Astro, astocyte; Oligo, oligodendrocyte. 
Table 1: Genes comprising the 25-core signature in all optic glioma models

\begin{tabular}{|c|c|}
\hline Gene & Name \\
\hline Aldh1l1 & aldehyde dehyrogenase 1 family member L1 \\
\hline Btbd3 & BTB (POZ) domain-containing 3 \\
\hline Btbd17 & BTB (POZ) domain-containing 17 \\
\hline Cd38 & CD38 \\
\hline Cspg5 & chondroitin sulfate proteoglycan 5 (Caleb) \\
\hline Cxcl14 & C-X-C motif chemokine ligand 14 \\
\hline Elmo2 & engulfment and cell motility 2 \\
\hline Etv5 & ets variant $5($ Erm $)$ \\
\hline Fabp5 & fatty acid binding protein 5 (E-Fabp) \\
\hline Fgfrll & fibroblast growth factor receptor-like 1 \\
\hline Gjal & gap junction protein, alpha 1 (connexin-43) \\
\hline $\operatorname{Igdcc4}$ & immunoglobulin superfamily DCC subclass member 4 (Nope) \\
\hline Marcks & myristoylated alanine-rich protein kinase $\mathrm{C}$ substrate \\
\hline Ncan & neurocan $(\mathrm{Cspg} 3)$ \\
\hline Ntsr 2 & neurotensin receptor 2 \\
\hline$P c d h g c 3$ & protocadherin gamma subfamily C, 3 \\
\hline Pygm & glycogen phosphorylase, muscle associated \\
\hline S1prl & sphingosine-1-phosphate receptor 1 (Edg1) \\
\hline$S d c 3$ & syndecan 3 \\
\hline Sfxn5 & sideroflexin 5 \\
\hline Shc3 & SHC adaptor protein 3 (N-Shc) \\
\hline Slc6a11 & solute carrier family 6 member 11 (Gat3) \\
\hline Spry4 & sprouty homolog 4 \\
\hline Tril & TLR4 interactor with leucine-rich repeats \\
\hline$W n t 7 b$ & wingless-type MMPTV integration site family, member 7B \\
\hline
\end{tabular}

these tumors shared a core gene expression signature. In this regard, we identified a 25-gene signature that is universally differentially expressed (increased) relative to normal optic nerves across the 27 optic glioma specimens (Table 1; Figure 2A). Interestingly, this signature was most pronounced in the FMOC and FMC tumors and relatively diminished in the FMPC tumors. Accordingly, our deconvolution model suggests that these genes are predominantly associated with OPC-like cells. While there are more $\mathrm{GFAP}^{+}$cells in all $\mathrm{Nfl}$ optic glioma models relative to the non-neoplastic optic nerve, there are only small differences in the relative proportions of Olig2 $2^{+}$, $\mathrm{GFAP}^{+}$, or $\mathrm{NG}^{+}$glial cells between the various GEM models ([24, 25]; Supplementary Figure 1). In addition, GO-term analysis of the core genes did not reveal enrichment in specific categories (data not shown). As before, we favor the interpretation that this core signature represents a "neoplastic state" gene expression network established by the glioma ecosystem. Since this core gene expression signature distinguishes normal from tumorbearing optic nerve, we hypothesize that it might be used to identify brain tumor treatments that best normalize the glioma ecosystem.

To explore this idea further, we leveraged prior preclinical studies from our laboratory in which conventional and biologically-targeted chemotherapy agents were evaluated prior to clinical translation. While our previous preclinical experiments demonstrated that temozolomide (TMZ) is effective at inducing tumor death [29], we now employed carboplatin as a standard chemotherapy reference, as it is the first line therapy for children with NF1-OPG [30, 31]. Similar to that observed in FMC mice following TMZ treatment, carboplatin induced a significant reduction in both tumor volume and proliferation ( $\% \mathrm{Ki} 67^{+}$cells; Figure 2B). Additionally, we evaluated the effect of two biologicallytargeted therapies previously shown to inhibit mouse $N f 1$ optic glioma growth in vivo. We selected rapamycin (mechanistic target of rapamycin [mTOR] inhibitor; [29]) and minocycline (microglia inhibitor; [17]) to inhibit neoplastic and stromal cell function, respectively, in separate experiments. 
Overall, we found that rapamycin treatment resulted in very few changes in the 25 -core gene expression profile compared to carboplatin and minocycline. This result is not surprising in light of recent clinical studies on a related NF1-associated benign tumor, the plexiform neurofibroma, in which rapamycin analogs exhibited little clinical efficacy [32, 33]. Importantly, while carboplatin treatment clearly inhibited tumor growth in vivo, there was also little effect on the 25-core gene expression signature. This finding suggests that this time-tested chemotherapy may not normalize the tumor ecosystem. In this regard, tumor shrinkage is observed in the minority of children with NF1-OPG [34, 35], and, when observed, is often relatively modest or not longstanding (durable response) [36-38].

While neither anti-neoplastic cell therapy normalized the optic glioma core signature, minocycline treatment resulted in downregulation of this 25-core gene signature in the majority of mice (Figure 2C). Furthermore, analysis of the entire set of genes that were normalized by minocycline treatment using our deconvolution model showed that these alterations were largely associated with OPC-like cells, rather than microglia (Figure 2D). These results imply that blocking microglia function with minocycline has its greatest impact on the tumors themselves. This finding is consistent with recent RNAsequencing studies on Nf1 optic glioma-associated microglia, in which one chemokine (CCL5) made by these microglia positively regulates both microglia recruitment and glioma growth [19]. Future studies aimed at normalizing the tumor microenvironment using stroma-targeted treatments may prove most effective at reestablishing a normal tissue ecosystem, and result in more durable glioma responses in human clinical trials.

\section{A PTEN/PI3K-dependent gene signature is associated with poor glioma prognosis}

As noted above, Pten deletion altered both the lineage resemblance and core signature expression of the FMC optic glioma model. Heat map representation

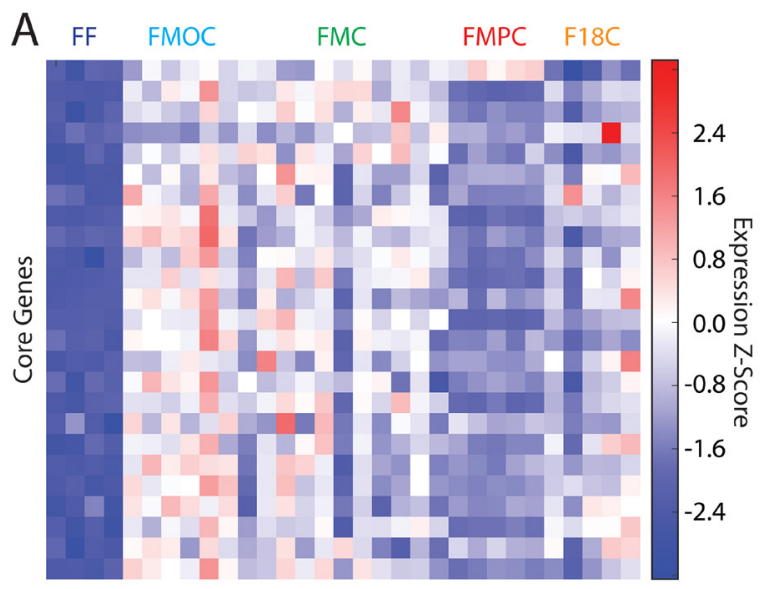

B
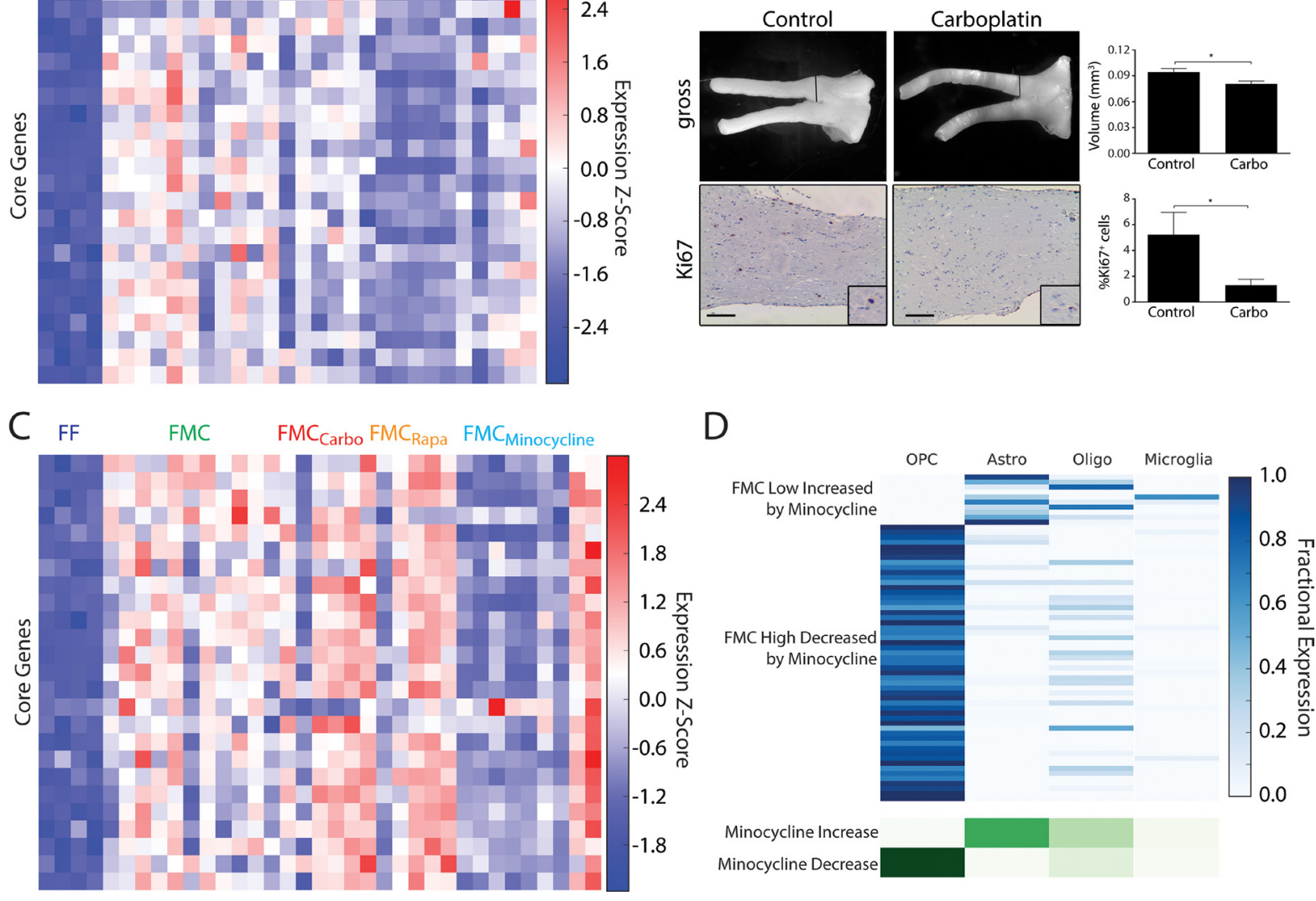

Figure 2: (A) Heatmap of RNA-Seq expression z-scores computed for the 25-gene core signature that is commonly differentially upregulated across all optic glioma models. (B) FMC mice treated with carboplatin at 3 months of age, and analyzed 1 month later, reveals decreased tumor volumes and percentages of $\mathrm{Ki}_{6} 7^{+}$cells. Control mice were treated with vehicle only and processed identically. ${ }^{*} p<0.05$. Scale bar, $100 \mu \mathrm{m}$. (C) Same as (A), but for minocycline-treated and untreated FMC gliomas and FF controls. (D) Deconvolution of genes that are normalized in FMC by minocycline (highly or lowly expressed in FMC gliomas vs. FF controls, but either decreased or increased, respectively, by minocycline treatment). 
(Figure 3A) and deconvolution profiles (Figure 3B) revealed a differentially-expressed gene signature between FMC and FMPC specimens (Table 2). In the latter analysis, genes associated with the FMC and FMPC models were enriched in OPC-like and astrocytic gene expression, respectively. Importantly, these differentially-expressed signatures were not reflected in the relative abundance of either OPCs or astrocytes (Supplementary Figure 1).

Because PTEN is a negative regulator of PI3K/ AKT signaling and Nfl optic glioma growth [25] we hypothesized that pharmacologic inhibition of PI3K might normalize this differentially-expressed gene signature in FMPC mice. The ability of a PI3K-specific inhibitor, BKM120, to restore the FMC gene expression pattern in FMPC mice was revealed by sample-specific and average expression profiles (Figure 4A, 4B). Consistent with differential expression of OPC-associated genes in FMC tumors, deconvolution demonstrated a pronounced enrichment of OPC-associated genes in FMPC tumors following treatment with BKM120 (Figure 4C). These results demonstrate that a PI3K-regulated gene signature (Table 3) partly accounts for the differences observed between FMC and FMPC optic gliomas.

Since the heterozygous Pten deletion was restricted to the neoplastic cells by virtue of the fact that Cre-mediated Pten reduction was targeted to GFAP transgene-expressing cells containing somatic $N f 1$ loss, we sought to determine whether this signature was maintained in optic glioma stem cells (o-GSCs) that derived from FMC and FMPC mouse tumors. Previously, we showed that o-GSCs from FMC mice are true cancer stem cells, with the ability to self-renew, undergo multi-lineage differentiation, (Supplementary Figure 2) and form glioma-like lesions when transplanted into naïve mice [39]. RNA-seq was performed using independent isolates of o-GSCs from at least three pools of FMC and FMPC mice $(n=2$ mice/ pool). Similar to that observed in the whole tumors, there was a relative depletion of the BKM120 normalized signature in FMC versus FMPC o-GSCs (Figure 4D), supporting a primary effect of heterozygous Pten deletion on the neoplastic cells in these gliomas. In addition, to determine whether BKM120 treatment normalizes this gene signature in purified o-GSCs, we treated separate pools of o-GSCs derived from FMPC mice with BKM120 in vitro and observed decreased PI3K/AKT activity (Supplementary Figure 3). As observed following BKM120 treatment of FMPC mice, we found the same signature to be significantly depleted in BKM120 treated o-GSCs relative to vehicletreated controls (Figure 4E). Taken together, the BKM120normalized gene signature behaves similarly in purified glioma stem cells and glioma tissue from the same models.

One of the clinically-relevant uses of these differentially-expressed gene signatures is their application to predicting survival in patients with gliomas. We first examined the 25-gene core signature, common to all murine $\mathrm{Nfl}$ optic gliomas, and found that it did not separate patients with either low-grade or high-grade gliomas into clinically-relevant subgroups based on overall survival or progression-free survival (data not shown). However, in striking contrast, differences in patient survival were observed using an expression signature comprised of the top 50 FMPC-associated genes that were most significantly normalized by BKM120 treatment (BKM120 normalized signature). For these analyses, gene set enrichment analysis (GSEA) was employed to assess the relative expression of this gene signature across human
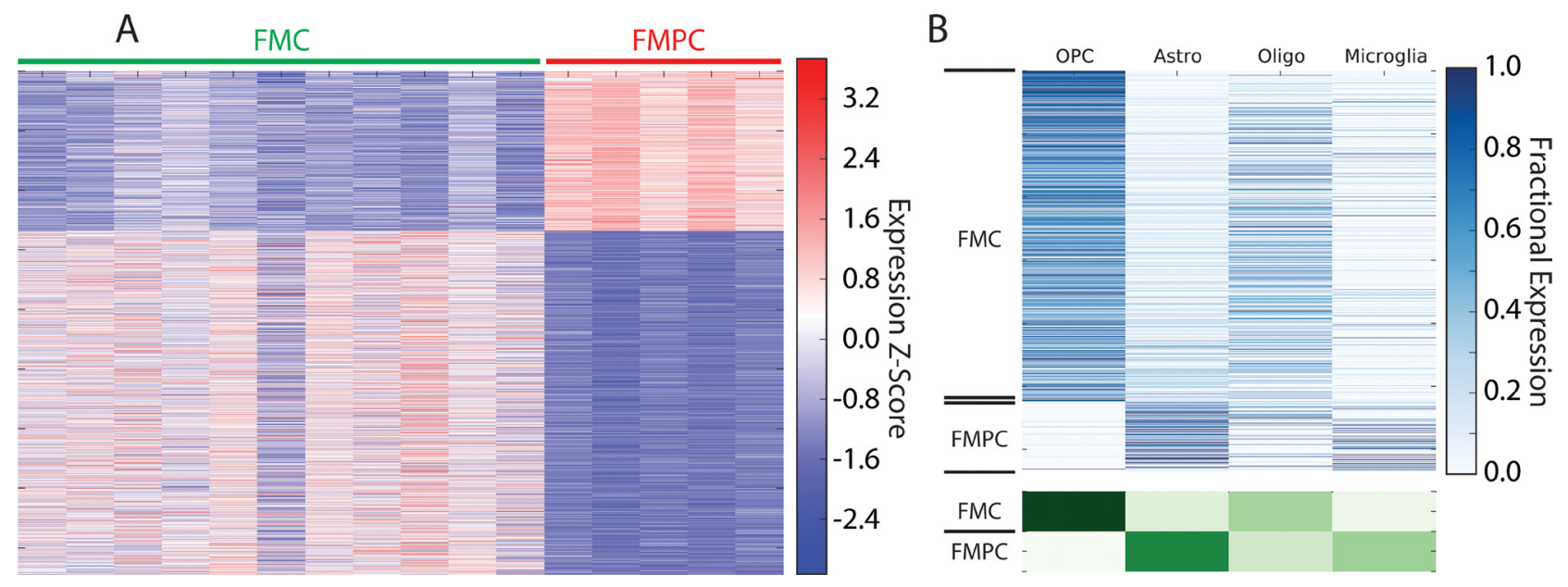

Figure 3: (A) Heatmap of RNA-Seq expression z-scores computed for genes that are differentially expressed $\left(p_{\text {adj }}<0.01, \mid \log _{2}(\right.$ foldchange $\mid>1$ ) between FMPC and FMC gliomas. (B) Deconvolution of genes that are differentially expressed between FMPC and FMC gliomas. In the top panel, each row in the heatmap corresponds to a specific differentially expressed gene and in each column corresponds to a cell type. The lower panel shows the aggregate expression in each cell type for all genes that are high in FMPC or FMC. The analysis shows that the FMC tumors are highly enriched in OPC-like gene expression compared to FMPC tumors, which are highly enriched in astrocytic gene expression. 
Table 2: Genes differentially-expressed between FMC and FMPC optic gliomas

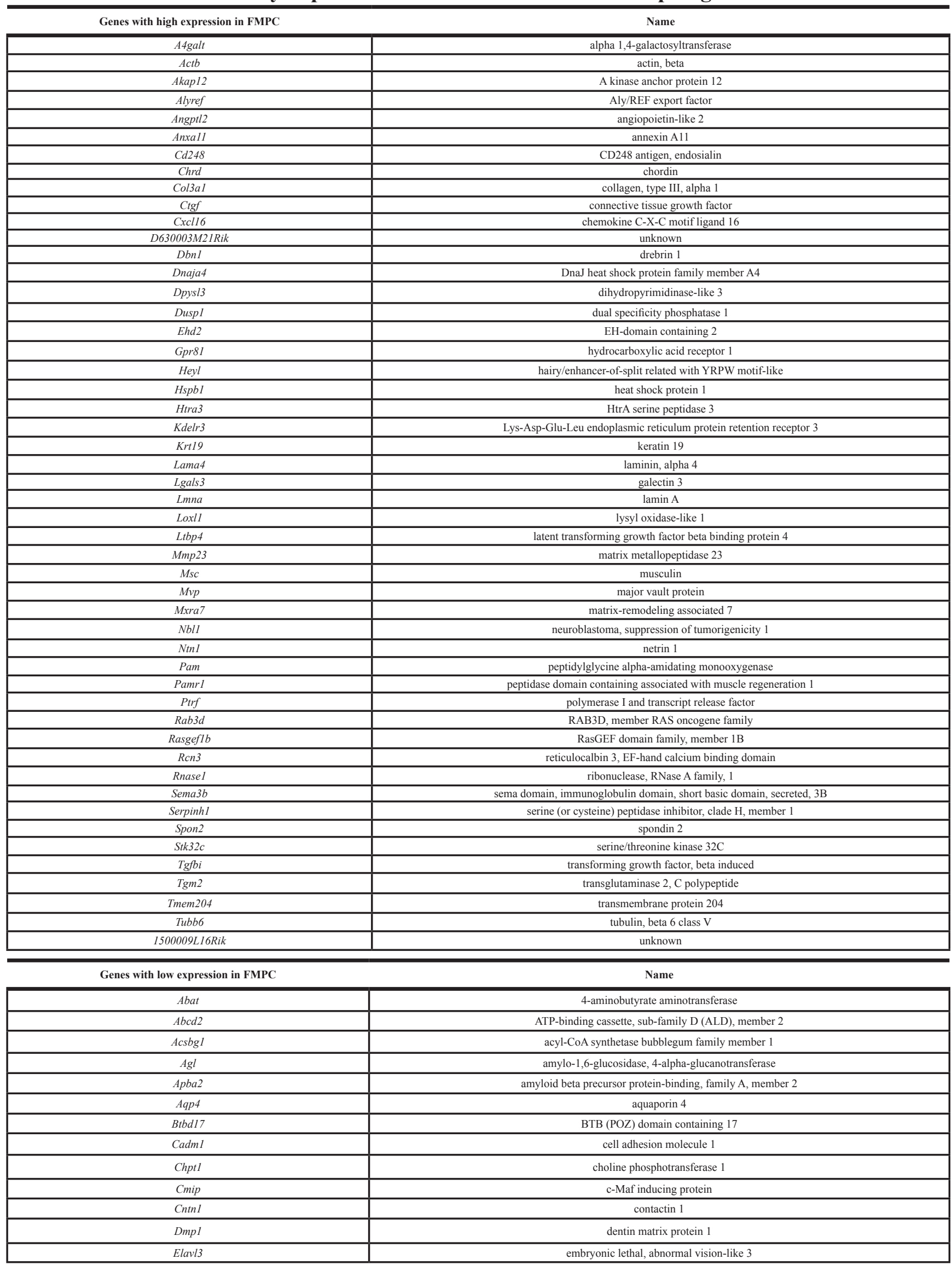




\begin{tabular}{|c|c|}
\hline Elovl2 & elongation of very long chain fatty acids-like 2 \\
\hline Elovl6 & ELOVL family member 6 , elongation of long chain fatty acids \\
\hline Frrs1l & ferric-chelate reductase 1 like \\
\hline Fut9 & fucosyltransferase 9 \\
\hline Gabrbl & gamma-aminobutyric acid A receptor, subunit beta 1 \\
\hline Gnaq & guanine nucleotide binding protein, alpha q polypeptide \\
\hline Gria 2 & glutamate receptor, ionotropic, AMPA2 \\
\hline Gria4 & glutamate receptor, ionotropic, AMPA4 \\
\hline Grin $3 a$ & glutamate receptor ionotropic, NMDA3A \\
\hline Gyk & glycerol kinase \\
\hline$H s d 11 b 1$ & hydroxysteroid 11-beta dehydrogenase 1 \\
\hline $\operatorname{Itgb8}$ & integrin beta 8 \\
\hline Kcna2 & potassium voltage-gated channel, shaker-related subfamily, member 2 \\
\hline KcnkI & potassium channel, subfamily $\mathrm{K}$, member 1 \\
\hline Luzp2 & leucine zipper protein 2 \\
\hline Mdga2 & MAM domain containing glycosylphosphatidylinositol anchor 2 \\
\hline Ncan & neurocan \\
\hline Negrl & neuronal growth regulator 1 \\
\hline Nfia & nuclear factor $\mathrm{I} / \mathrm{A}$ \\
\hline Nfib & nuclear factor $\mathrm{I} / \mathrm{B}$ \\
\hline Nlgnl & neuroligin 1 \\
\hline Nrxnl & neurexin I \\
\hline Paqr8 & progestin and adipoQ receptor family member VIII \\
\hline Pcdh15 & protocadherin 15 \\
\hline Phkal & phosphorylase kinase alpha 1 \\
\hline Prex2 & phosphatidylinositol-3,4,5-trisphosphate-dependent Rac exchange factor 2 \\
\hline Ptprd & protein tyrosine phosphatase, receptor type, D \\
\hline PtprzI & protein tyrosine phosphatase, receptor type $Z$, polypeptide 1 \\
\hline $\operatorname{Rgs} 7 b p$ & regulator of G-protein signalling 7 binding protein \\
\hline Slcla2 & solute carrier family 1, member 2 \\
\hline Slc4a4 & solute carrier family 4 , member 4 \\
\hline Spag5 & sperm associated antigen 5 \\
\hline Timp4 & tissue inhibitor of metalloproteinase 4 \\
\hline Tmed5 & transmembrane p24 trafficking protein 5 \\
\hline Tmem $132 b$ & transmembrane protein $132 \mathrm{~B}$ \\
\hline Tmtc2 & transmembrane and tetratricopeptide repeat containing 2 \\
\hline Tshzl & teashirt zinc finger family member 1 \\
\hline
\end{tabular}

low- and high-grade glioma surgical specimens collected and profiled by The Cancer Genome Atlas (TCGA) consortium (obtained from the Broad Institute GDAC Firehose site). In both low-grade (Figure 5A) and highgrade (Figure 5B) glioma, patients with tumors depleted in the BKM120 treatment signature exhibited significantly enhanced progression-free survival relative to those with a PTEN-enriched signature.

Taken together, these data support a model in which murine $\mathrm{Nfl}$ optic gliomas operate as an ecosystem composed of a diverse collection of cellular and acellular elements. The observation that the discovered 25-core differential gene expression pattern, which distinguishes tumor from non-neoplastic tissue, does not emanate from a single cell type (o-GSCs or microglia) argues that it represents networks of genes that are differentially expressed as a result of forming a tumor. This new ground state thus defines glioma at a molecular level, independent of gross morphological changes or alterations in cell composition or proliferation. Moreover, the established glioma gene expression profile provides a platform to evaluate potential chemotherapeutic agents, either alone or in combination, for their potential to normalize the tumor ecosystem and achieve greater treatment responses. In addition, co-existing genetic mutations targeted to the neoplastic cell compartment (Pten and NfI inactivation) can create differential core signatures that reflect the individual contributions of these molecular events to the cancer cells, which can be used to identify prognostic signatures relevant to patient survival and the potential interpretation of successful inhibition of the regulated growth control pathways. Future studies employing these types of approaches may be instructive, not only for defining what constitutes tumor, but also for identifying a possible Achilles heel network node for therapeutic targeting.

\section{MATERIALS AND METHODS}

\section{Mice}

FF $\left(N f 1^{\text {flox/flox }}\right)$, FMC (Nf1 flox/mut; GFAP-Cre $)$ FMPC $\left(N f 1^{\text {flox/mut; }}\right.$ Pten ${ }^{\text {flox/wt; }}$ GFAP-Cre), F18C (Nf1 gene mutation c.2041C $>$ T; p.R681X; Nf1 $1^{\text {flox/R681*}}$; GFAP-Cre) mice were generated as previously described [23-25]. FMOC mice were generated by intercrossing $\mathrm{Nflflox} /$ mut mice with Olig2-Cre mice (B6-Olig2 $2^{\mathrm{tm} 2}$ (TVA, cre)Rth/J; Jackson Laboratory; $N f 1^{\text {flox/mut; }}$ Olig2-Cre). All mice were maintained on a $\mathrm{C} 57 \mathrm{Bl} / 6$ background and procedures performed in accordance with an approved Animal Studies Committee protocol at Washington University. 


\section{Pharmacological studies}

$50 \mathrm{mg} / \mathrm{kg}$ /day minocycline (Sigma-Aldrich; PBS, vehicle) was delivered by intraperitoneal (i.p.) injection 5 days/week for two weeks [17]. $5 \mathrm{mg} / \mathrm{kg} /$ day rapamycin (Selleckchem; 5.2\% Tween 80/5.2\% polyethylene glycol 400 as vehicle) was administered by i.p. injection 5 days/ week for two weeks [29]. $20 \mathrm{mg} / \mathrm{kg} /$ day NVP-BKM120 (Selleckchem; N-methyl-2-pyrrolidone/ polyethylene glycol $300(10 / 90 \mathrm{v} / \mathrm{v})$ as vehicle) was delivered by oral gavage 5 days/week for two weeks [40]. $60 \mathrm{mg} /$ $\mathrm{kg} /$ day carboplatin (Sigma-Aldrich; PBS as vehicle) was administered by i.p. injection every other day for 1 week. All optic nerves were harvested two weeks after the initial injection, and processed for RNAseq, immunohistochemistry and optic nerve measurements as described below.

\section{Optic nerve measurements}

Following perfusion with Ringers solution and 4\% paraformaldehyde (PFA), optic nerves were photographed and volumes measured as previously described [29].

\section{Immunohistochemistry}

Optic nerves were microdissected and processed as described previously [41]. Antibodies used are listed in Supplementary Table 1.

\section{Cell culture}

Optic glioma stem cells (o-GSCs) were isolated and maintained as previously described [39]. For NVPBKM120 treatments, cells were cultured in neural stem
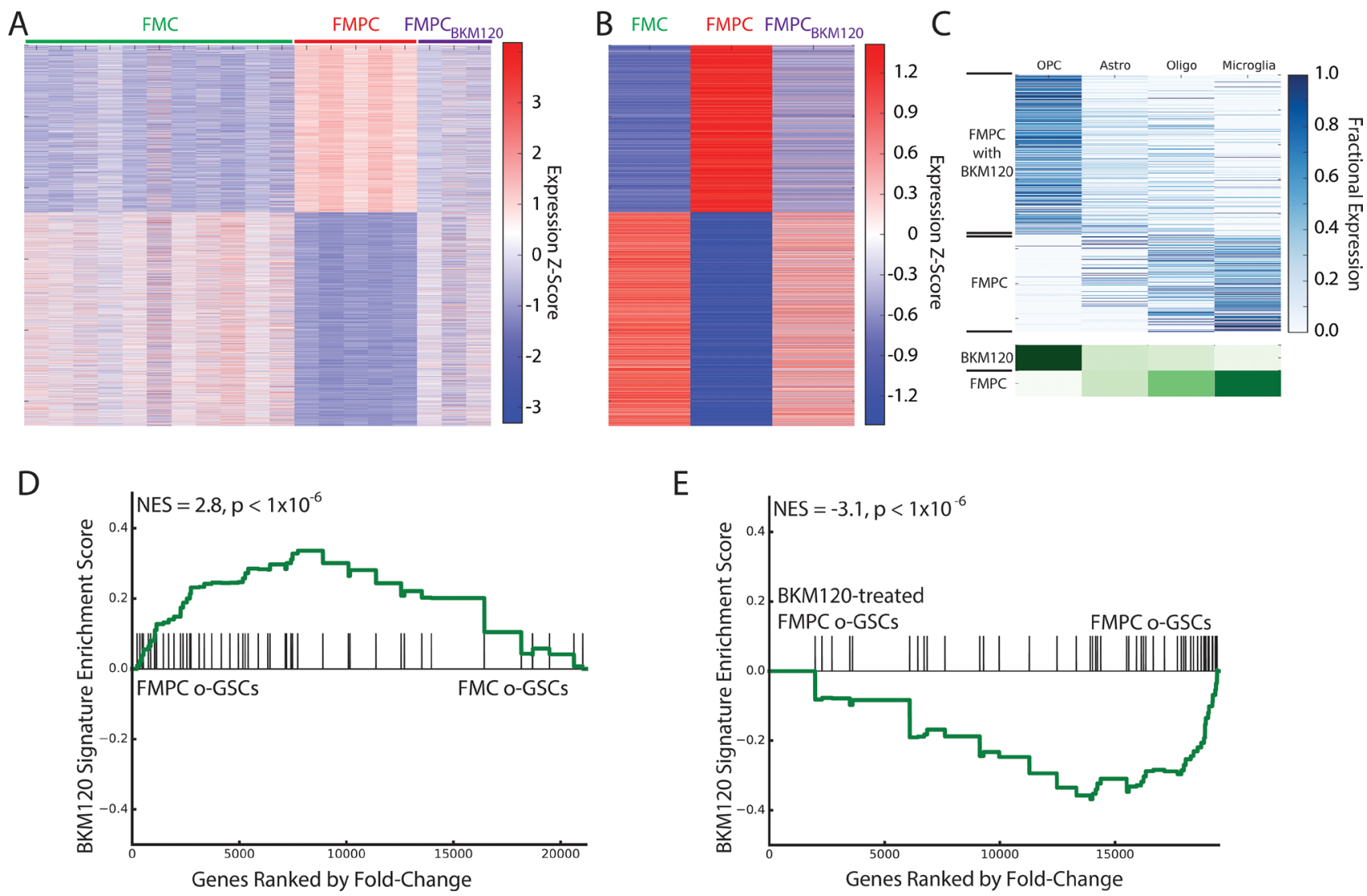

Figure 4: (A) Heatmap of RNA-Seq expression z-scores computed for genes that are differentially expressed $\left(p_{\text {adj }}<0.01, \mid \log _{2}(\right.$ foldchange) $\mid>1$ ) between FMPC and FMC gliomas across FMC, FMPC, and BKM120-treated FMPC gliomas. Many of the differentially expressed genes are normalized by BKM120 treatment. (B) Same as (A), but averaged over all samples for each group. (C) Deconvolution of genes that are differentially expressed between BKM120-treated and untreated FMPC gliomas. In the top panel, each row in the heatmap corresponds to a specific differentially-expressed gene and in each column corresponds to a cell type. The lower panel shows the aggregate expression in each cell type for all genes that are high in BKM120-treated and untreated FMPC tumors. The analysis shows that the treated tumors are highly enriched in OPC-like gene expression (similar to the FMC tumors) compared to the untreated tumors. (C) GSEA showing enrichment of the 50-gene BKM120 normalization signature in the RNA-Seq profile of FMPC- compared to FMC-derived o-GSCs. (D) GSEA showing depletion of the 50-gene BKM120 normalization signature in BKM120-treated FMPC-derived o-GSCs compared to vehicle-treated cells. 
Table 3: Genes comprising the 50-core signature responsive to BKM-120 inhibition

\begin{tabular}{|c|c|}
\hline Gene & Name \\
\hline$A c t b$ & actin, beta \\
\hline Akrlal & aldo-keto reductase family 1 member A1 \\
\hline Aldoa & aldolase, fructose-biphosphate A \\
\hline Alyref & Aly/REF export factor \\
\hline $\operatorname{Arf5}$ & ADP ribosylation factor 5 \\
\hline Arl4d & ADP ribosylation factor-like GTPase 4D \\
\hline Arpc3 & actin-related protein $2 / 3$ complex subunit 3 \\
\hline Atp6vlf & ATPase $\mathrm{H}+$ transporting $\mathrm{V} 1$ subunit $\mathrm{F}$ \\
\hline Cct5 & chaperonin-containing TCP1 subunit 5 \\
\hline$C d 81$ & CD81 antigen \\
\hline Cdc37 & cell division cycle 37 \\
\hline$C d r 1$ & cerebellar degeneration related protein 1 \\
\hline Cebpb & CCAAT/enhancer binding protein, beta \\
\hline Chp2 & calcineurin-like EF-hand protein 2 \\
\hline Cilp & cartilage intermediate layer protein \\
\hline Dpysl2 & dihydropyrimidinase like 2 \\
\hline Duspl & dual specificity phosphatase 1 \\
\hline Dynll1 & dynein light chain LC8-type 1 \\
\hline Eif3d & eukaryotic translation initiation factor 3 subunit D \\
\hline Ftl1 & ferritin light polypeptide 1 \\
\hline Fxyd1 & FXYD domain-containing ion transport regulator 1 \\
\hline Gpr81 & hydroxycarboxylic acid receptor 1 \\
\hline Herpud1 & homocysteine inducible ER protein with ubiquitin-like domain 1 \\
\hline Hnrnpl & heterogeneous nuclear ribonucleoprotein \\
\hline Ifitm2 & interferon-induced transmembrane protein 2 \\
\hline $\operatorname{Irf} 2 b p 2$ & interferon regulatory factor 2 binding protein 2 \\
\hline Kdelr3 & KDEL endoplasmic reticulum protein retention receptor 3 \\
\hline Loxl1 & lysyl oxidase like 1 \\
\hline Lsp1 & lymphocyte-specific protein 1 \\
\hline Map1lc3b & microtubule-associated protein 1 light chain 3 beta \\
\hline Mrpl12 & mitochondrial ribosomal protein L12 \\
\hline Oat & ornithine aminotransferase \\
\hline Opalin & oligodendrocytic myelin paranodal and inner loop protein \\
\hline Pam & peptidylglycine alpha-amidating monooxygenase \\
\hline Plat & plasminogen activator, tissue type \\
\hline Plekhf1 & pleckstrin homology and FYVE domain containing 1 \\
\hline Rasgeflb & RasGEF domain family member $1 \mathrm{~B}$ \\
\hline Rhoc & ras homolog family member $\mathrm{C}$ \\
\hline Slc6a13 & solute carrier family 6 member 13 \\
\hline Snrpb & small ribonuclear ribonucleoprotein polypeptides B and B1 \\
\hline Spon2 & spondin 2 \\
\hline Sqstm 1 & sequestosome 1 \\
\hline$T g f b i$ & transforming growth factor, beta-induced \\
\hline
\end{tabular}




\begin{tabular}{|c|c|}
\hline Tppp3 & tubulin polymerization promoting protein family member 3 \\
\hline Tram1 & translocation-associated membrane protein 1 \\
\hline Ubb & ubiquitin B \\
\hline Vtn & vitronectin \\
\hline Wdr1 & WD repeat domain 1 \\
\hline Ywhaz & tyrosine 3-monooxygenase/tryptophan 5-monooxygenase activation protein zeta \\
\hline $1810011 O 10 R i k$ & unknown \\
\hline
\end{tabular}

cell medium without EGF and bFGF for 1 hour, followed by treatment with DMSO (vehicle) or $0.2 \mu \mathrm{M}$ NVPBKM120 for 5 hours at $37^{\circ} \mathrm{C}$, and cells harvested for RNA isolation or western blotting.

\section{Western blotting}

Cells were lysed as described previously and western blotting performed using the antibodies listed in Supplementary Table $1[42,43]$.

\section{Immunocytochemistry}

o-GSCs were adhered to fibronectin $(50 \mu \mathrm{g} / \mathrm{ml}) /$ poly-D-lysine $(10 \mu \mathrm{g} / \mathrm{ml})$-coated surfaces and cultured in neural stem cell medium supplemented with $\mathrm{N} 2$,
B27, EGF and bFGF for 24 hours at $37^{\circ} \mathrm{C}$ and fixed with 4\% PFA for immunostaining. o-GSCs were also differentiated as previously described [39], fixed with $4 \%$ PFA, and immunostained with the antibodies listed in Supplementary Table 1.

\section{RNA extraction and mRNA isolation}

RNA was extracted from Trizol-preserved tissue and cell lysates using isopropanol precipitation. Each total RNA sample was re-suspended in $20 \mathrm{uL}$ of nuclease-free water supplemented with $0.5 \mathrm{U} / \mathrm{uL}$ RNase inhibitor (SUPERaseIN, Life Technologies). A 5'-biotinylated, LNA-enhanced oligo(dT) capture probe (Exiqon) was used to isolate poly $(\mathrm{A})+$ mRNA as described previously [44].
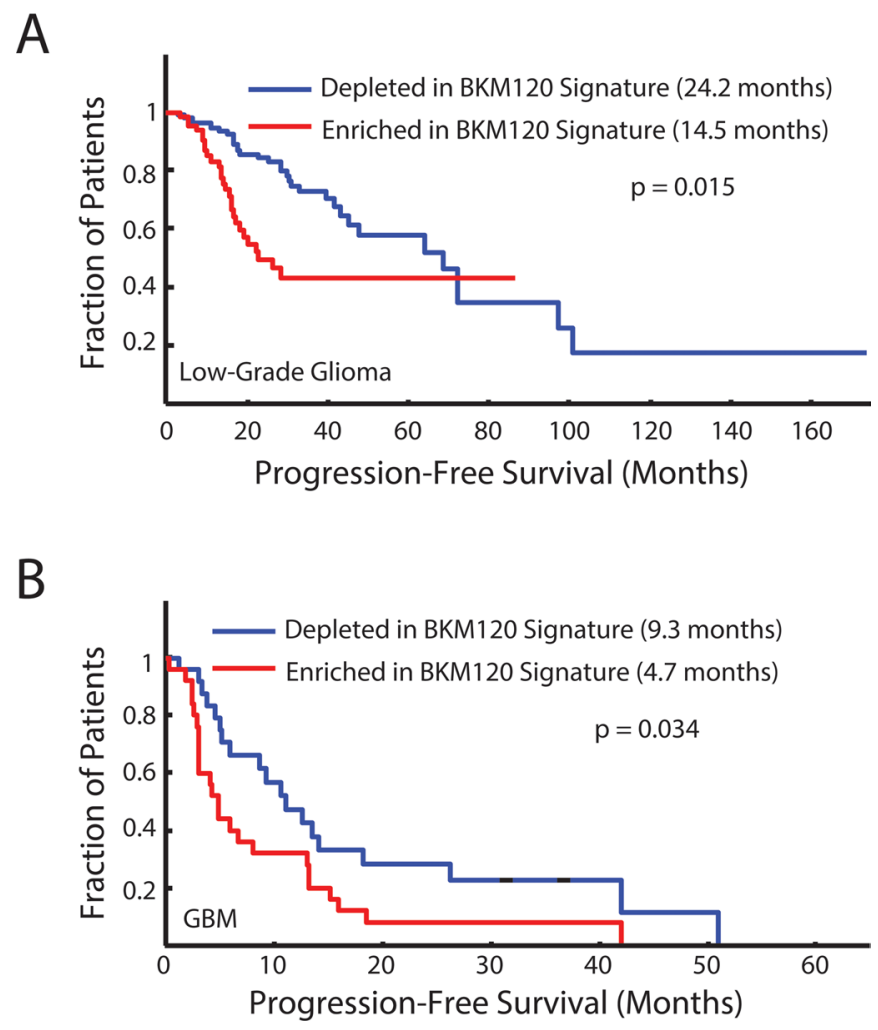

Figure 5: Progression-free survival curves for human low-grade gliomas (A) and glioblastomas (B) that are either highly enriched or depleted of the top 50 genes that are differentially expressed between FMPC and FMC gliomas and most significantly normalized by BKM120 treatment of FMPC gliomas. Depletion of the 50-gene BKM120 normalization signature is associated with better progressionfree survival in both low-grade $(p=0.015)$ and high-grade $(p=0.034)$ human brain tumors. 


\section{Library construction and sequencing}

RNA-Seq libraries were constructed using SMARTer Stranded RNA-Seq kit (Clontech Laboratories) according to the manufacturer's instructions. mRNA was fragmented for 4 minutes at $94^{\circ} \mathrm{C}$ and cDNA libraries were purified twice using AMPure XP beads (Beckman) prior to PCR enrichment. After PCR enrichment, the resulting sequencing libraries were quantified using a Bioanalyzer (Agilent) and Qubit (ThermoFisher). The libraries were pooled and sequenced on an Illumina NextSeq 500 using 75-cycle High Output Kits.

\section{RNA-Seq data processing}

Prior to sequence alignment, the first five nucleotides from the 5 '-end of each read were clipped to eliminate the GC-rich, template-switching sequence inserted during reverse transcription. The reads were aligned to the mouse genome and transcriptome (mm10, USCS annotation from Illumina iGenomes) using Tophat 2 [45]. HTSeq was used to quantify the number of reads associated with each gene and DESeq2 for differential expression analysis [46]. The RNA-seq data will be deposited in GEO.

\section{Deconvolution of cell type-specific gene expression and gene set enrichment analysis}

Computational deconvolution of cell type-specific gene expression was accomplished using the methods described in our previous studies [27, 28] with one improvement. As cell type-specific markers, we used Cspg4 (OPC), Gfap (astrocyte), Mog (oligodendrocyte), Rbfox3 (neuron), and Aifl (microglia). Gene set enrichment analysis was conducted using the GSEA software package available from the Broad Institute. For all of the GSEA presented here, we used pre-ranked/ classical mode to compute enrichment scores.

\section{ACKNOWLEDGMENTS}

We thank Courtney Corman for expert technical assistance.

\section{CONFLICTS OF INTEREST}

The authors declare no conflicts of interest.

\section{GRANT SUPPORT}

This work was supported by grants from the National Cancer Institute (U01-CA195692-01 to DHG), Department of Defense (W81XWH-13-1-0094 to DHG and PAS), and James S. McDonnell Foundation (to DHG and PAS).

\section{REFERENCES}

1. Hanahan D, Weinberg RA. Hallmarks of cancer: the next generation. Cell. 2011; 144:646-674.

2. Amend SR, Pienta KJ. Ecology meets cancer biology: the cancer swamp promotes the lethal cancer phenotype. Oncotarget. 2015; 6:9669-9678. doi: 10.18632/oncotarget.3430.

3. Bolouri H. Network dynamics in the tumor microenvironment. Semin Cancer Biol. 2015; 30:52-59.

4. Ryu D, Joung JG, Kim NK, Kim KT, Park WY. Deciphering intratumor heterogeneity using cancer genome analysis. Hum Genet. 2016; 135:635-642.

5. Gajewski TF, Schreiber H, Fu YX. Innate and adaptive immune cells in the tumor microenvironment. Nat Immunol. 2013; 14:1014-1022.

6. Quail DF, Joyce JA. Microenvironmental regulation of tumor progression and metastasis. Nat Med. 2013; 19:1423-1437.

7. Pyonteck SM, Akkari L, Schuhmacher AJ, Bowman RL, Sevenich L, Quail DF, Olson OC, Quick ML, Huse JT, Teijeiro V, Setty M, Leslie CS, Oei Y, et al. CSF-1R inhibition alters macrophage polarization and blocks glioma progression. Nat Med. 2013; 19:1264-1272.

8. a Dzaye OD, Hu F, Derkow K, Haage V, Euskirchen P, Harms C, Lehnardt S, Synowitz M, Wolf SA, Kettenmann H. Glioma Stem Cells but Not Bulk Glioma Cells Upregulate IL-6 Secretion in Microglia/ Brain Macrophages via Toll-like Receptor 4 Signaling. J Neuropathol Exp Neurol. 2016; 75:429-440.

9. Hu F, a Dzaye OD, Hahn A, Yu Y, Scavetta RJ, Dittmar G, Kaczmarek AK, Dunning KR, Ricciardelli C, Rinnenthal JL, Heppner FL, Lehnardt S, Synowitz M, et al. Glioma-derived versican promotes tumor expansion via glioma-associated microglial/macrophages Toll-like receptor 2 signaling. Neuro Oncol. 2015; 17:200-210.

10. $\mathrm{Hu}$ F, Ku MC, Markovic D, a Dzaye OD, Lehnardt S, Synowitz M, Wolf SA, Kettenmann H. Glioma-associated microglial MMP9 expression is upregulated by TLR2 signaling and sensitive to minocycline. Int J Cancer. 2014; 135:2569-2578.

11. Hambardzumyan D, Gutmann DH, Kettenmann H. The role of microglia and macrophages in glioma maintenance and progression. Nat Neurosci. 2015; 19:20-27.

12. Arcuri C, Fioretti B, Bianchi R, Mecca C, Tubaro C, Beccari T, Franciolini F, Giambanco I, Donato R. Microglia-glioma cross-talk: a two way approach to new strategies against glioma. Front Biosci (Landmark Ed). 2017; 22:268-309.

13. Shen X, Burguillos MA, Osman AM, Frijhoff J, CarrilloJimenez A, Kanatani S, Augsten M, Saidi D, Rodhe J, Kavanagh E, Rongvaux A, Rraklli V, Nyman U, et al. Glioma-induced inhibition of caspase-3 in microglia promotes a tumor-supportive phenotype. Nat Immunol. $2016 ; 17: 1282-1290$ 
14. Son B, Lee S, Youn H, Kim E, Kim W, Youn B. The role of tumor microenvironment in therapeutic resistance. Oncotarget. 2017; 8:3933-3945. doi: 10.18632/oncotarget.13907.

15. Junttila MR, de Sauvage FJ. Influence of tumour microenvironment heterogeneity on therapeutic response. Nature. 2013; 501:346-354.

16. Gutmann DH, McLellan MD, Hussain I, Wallis JW, Fulton LL, Fulton RS, Magrini V, Demeter R, Wylie T, Kandoth C, Leonard JR, Guha A, Miller CA, et al. Somatic neurofibromatosis type 1 (NF1) inactivation characterizes NF1-associated pilocytic astrocytoma. Genome Res. 2013; 23:431-439.

17. Daginakatte GC, Gutmann DH. Neurofibromatosis-1 (Nf1) heterozygous brain microglia elaborate paracrine factors that promote Nf1-deficient astrocyte and glioma growth. Hum Mol Genet. 2007; 16:1098-1112.

18. Daginakatte GC, Gianino SM, Zhao NW, Parsadanian AS, Gutmann DH. Increased c-Jun-NH2-kinase signaling in neurofibromatosis-1 heterozygous microglia drives microglia activation and promotes optic glioma proliferation. Cancer Res. 2008; 68:10358-10366.

19. Solga AC, Pong WW, Kim KY, Cimino PJ, Toonen JA, Walker J, Wylie T, Magrini V, Griffith M, Griffith OL, Ly A, Ellisman MH, Mardis ER, et al. RNA Sequencing of Tumor-Associated Microglia Reveals Ccl5 as a Stromal Chemokine Critical for Neurofibromatosis-1 Glioma Growth. Neoplasia. 2015; 17:776-788.

20. Pong WW, Higer SB, Gianino SM, Emnett RJ, Gutmann DH. Reduced microglial CX3CR1 expression delays neurofibromatosis-1 glioma formation. Ann Neurol. 2013; 73:303-308.

21. Yang FC, Ingram DA, Chen S, Zhu Y, Yuan J, Li X, Yang X, Knowles S, Horn W, Li Y, Zhang S, Yang Y, Vakili ST, et al. Nf1-dependent tumors require a microenvironment containing Nf1+/ - and c-kit-dependent bone marrow. Cell. 2008; 135:437-448.

22. Staser K, Yang FC, Clapp DW. Mast cells and the neurofibroma microenvironment. Blood. 2010; 116:157-164.

23. Bajenaru ML, Hernandez MR, Perry A, Zhu Y, Parada LF, Garbow JR, Gutmann DH. Optic nerve glioma in mice requires astrocyte $\mathrm{Nf1}$ gene inactivation and $\mathrm{Nf1}$ brain heterozygosity. Cancer Res. 2003; 63:8573-8577.

24. Toonen JA, Anastasaki C, Smithson LJ, Gianino SM, Li K, Kesterson RA, Gutmann DH. NF1 germline mutation differentially dictates optic glioma formation and growth in neurofibromatosis-1. Hum Mol Genet. (in press).

25. Kaul A, Toonen JA, Gianino SM, Gutmann DH. The impact of coexisting genetic mutations on murine optic glioma biology. Neuro Oncol. 2015; 17:670-677.

26. Rodriguez EF, Scheithauer BW, Giannini C, Rynearson A, Cen L, Hoesley B, Gilmer-Flynn H, Sarkaria JN, Jenkins S, Long J, Rodriguez FJ. PI3K/AKT pathway alterations are associated with clinically aggressive and histologically anaplastic subsets of pilocytic astrocytoma. Acta Neuropathol. 2011; 121:407-420.

27. Gill BJ, Pisapia DJ, Malone HR, Goldstein H, Lei L, Sonabend A, Yun J, Samanamud J, Sims JS, Banu M, Dovas A, Teich AF, Sheth SA, et al. MRI-localized biopsies reveal subtype-specific differences in molecular and cellular composition at the margins of glioblastoma. Proc Natl Acad Sci USA. 2014; 111:12550-12555.

28. Gonzalez C, Sims JS, Hornstein N, Mela A, Garcia F, Lei L, Gass DA, Amendolara B, Bruce JN, Canoll P, Sims PA. Ribosome profiling reveals a cell-type-specific translational landscape in brain tumors. J Neurosci. 2014; 34:10924-10936.

29. Hegedus B, Banerjee D, Yeh TH, Rothermich S, Perry A, Rubin JB, Garbow JR, Gutmann DH. Preclinical cancer therapy in a mouse model of neurofibromatosis-1 optic glioma. Cancer Res. 2008; 68:1520-1528.

30. Packer RJ, Ater J, Allen J, Phillips P, Geyer R, Nicholson HS, Jakacki R, Kurczynski E, Needle M, Finlay J, Reaman G, Boyett JM. Carboplatin and vincristine chemotherapy for children with newly diagnosed progressive low-grade gliomas. J Neurosurg. 1997; 86:747-754.

31. Packer RJ, Lange B, Ater J, Nicholson HS, Allen J, Walker R, Prados M, Jakacki R, Reaman G, Needles MN, et al. Carboplatin and vincristine for recurrent and newly diagnosed low-grade gliomas of childhood. J Clin Oncol. 1993; 11:850-856.

32. Weiss B, Widemann BC, Wolters P, Dombi E, Vinks A, Cantor A, Perentesis J, Schorry E, Ullrich N, Gutmann DH, Tonsgard J, Viskochil D, Korf B, et al. Sirolimus for progressive neurofibromatosis type 1-associated plexiform neurofibromas: a neurofibromatosis Clinical Trials Consortium phase II study. Neuro Oncol. 2015; 17:596-603.

33. Weiss B, Widemann BC, Wolters P, Dombi E, Vinks AA, Cantor A, Korf B, Perentesis J, Gutmann DH, Schorry E, Packer R, Fisher MJ. Sirolimus for non-progressive NF1associated plexiform neurofibromas: an NF clinical trials consortium phase II study. Pediatr Blood Cancer. 2014; 61:982-986.

34. Dodgshun AJ, Elder JE, Hansford JR, Sullivan MJ. Longterm visual outcome after chemotherapy for optic pathway glioma in children: Site and age are strongly predictive. Cancer. 2015; 121:4190-4196.

35. Dalla Via P, Opocher E, Pinello ML, Calderone M, Viscardi E, Clementi M, Battistella PA, Laverda AM, Da Dalt L, Perilongo G. Visual outcome of a cohort of children with neurofibromatosis type 1 and optic pathway glioma followed by a pediatric neuro-oncology program. Neuro Oncol. 2007; 9:430-437.

36. Shofty B, Mauda-Havakuk M, Weizman L, Constantini S, Ben-Bashat D, Dvir R, Pratt LT, Joskowicz L, Kesler A, Yalon M, Ravid L, Ben-Sira L. The effect of chemotherapy on optic pathway gliomas and their sub-components: A volumetric MR analysis study. Pediatr Blood Cancer. 2015; 62:1353-1359. 
37. Shofty B, Ben-Sira L, Freedman S, Yalon M, Dvir R, Weintraub M, Toledano H, Constantini S, Kesler A. Visual outcome following chemotherapy for progressive optic pathway gliomas. Pediatr Blood Cancer. 2011; 57:481-485.

38. Mahoney DH Jr, Cohen ME, Friedman HS, Kepner JL, Gemer L, Langston JW, James HE, Duffner PK, Kun LE. Carboplatin is effective therapy for young children with progressive optic pathway tumors: a Pediatric Oncology Group phase II study. Neuro Oncol. 2000; 2:213-220.

39. Chen YH, McGowan LD, Cimino PJ, Dahiya S, Leonard JR, Lee da Y, Gutmann DH. Mouse low-grade gliomas contain cancer stem cells with unique molecular and functional properties. Cell Rep. 2015; 10:1899-1912.

40. Kaul A, Toonen JA, Cimino PJ, Gianino SM, Gutmann DH. Akt- or MEK-mediated mTOR inhibition suppresses Nf1 optic glioma growth. Neuro Oncol. 2015; 17:843-853.

41. Hegedus B, Yeh TH, Lee DY, Emnett RJ, Li J, Gutmann DH. Neurofibromin regulates somatic growth through the hypothalamic-pituitary axis. Hum Mol Genet. 2008; 17:2956-2966.
42. Apicelli AJ, Uhlmann EJ, Baldwin RL, Ding H, Nagy A, Guha A, Gutmann DH. Role of the Rap1 GTPase in astrocyte growth regulation. Glia. 2003; 42:225-234.

43. Dasgupta B, Li W, Perry A, Gutmann DH. Glioma formation in neurofibromatosis 1 reflects preferential activation of K-RAS in astrocytes. Cancer Res. 2005; 65:236-245.

44. Pulko V, Davies JS, Martinez C, Lanteri MC, Busch MP, Diamond MS, Knox K, Bush EC, Sims PA, Sinari S, Billheimer D, Haddad EK, Murray KO, et al. Human memory $\mathrm{T}$ cells with a naive phenotype accumulate with aging and respond to persistent viruses. Nat Immunol. 2016; 17:966-975.

45. Kim D, Pertea G, Trapnell C, Pimentel H, Kelley R, Salzberg SL. TopHat2: accurate alignment of transcriptomes in the presence of insertions, deletions and gene fusions. Genome Biol. 2013; 14:R36.

46. Love MI, Huber W, Anders S. Moderated estimation of fold change and dispersion for RNA-seq data with DESeq2. Genome Biol. 2014; 15:550. 